\title{
TRAJECTOIRES ACADÉMIQUES ET FACTEURS PSYCHOSOCIAUX LIÉS À LA RÉUSSITE : LE RÔLE DE LA RÉSILIENCE DEPUIS UNE APPROCHE SYSTÉMIQUE
}

\author{
Author(s) / Auteur(s) : \\ Miriam APARICIO \\ Dr. en Education, UCA, Argentine (1995) \\ Dr. en Sciences de l'éducation, Paris V, René Descartes, Sorbonne| \\ Université en Argentine: Nacional de Cuyo (Professeur des universités) \\ CONICET (Conseil national pour la recherche scientifique, Argentine) - Chercheure supérieure \\ miriamapar@yahoo.com
}

\begin{abstract}
Résumé :
Cette recherche est inscrite dans la problématique générale de la Qualité de l'Université. Elle a été effectuée sur un échantillon des sujets qui rallongent leurs études de 6 facultés dans sa première instance (229 sujets; 17 cohortes, en continuant jusqu'au nos jours). L'objectif a été de connaître les variables/ dimensions associés à la réussite / échec universitaire. Dans cet article nous abordons la variable Résilience, en remarquant les différences par rapport à la Résistance. L'hypothèse générale est: une haute résilience (RESIL) agit positivement sur la réussite universitaire (RU). La méthodologie a été quantitative-qualitative. Le modèle intègre des variables de base, pédagogico-institutionnelles, structurelles et psychosociales. Parmi ces dernières, nous nous centrons dans cet article sur la Résilience en relation avec le niveau d'échec relatif universitaire.

En ce qui concerne les techniques utilisées (les plus cités dans la littérature internationale), nous avons appliqué une enquête semi-structurée et des tests spécifiques. Pour mesurer les variables psychosociales en relation la réussiteléchec universitaire (RU), les techniques ont été: les Stratégies pour "faire face » aux difficultés (Frydenberg \& Lewis, 1996), les styles attributionnels (Seligman, 1991), les facteurs motivationnels (Montero \& Alonso Tapia, 1992) et la Résilience (Henderson \& Milstein, 2003). Cette dernière technique est composée de 3 échelles et 6 sous-échelles qui montrent bien les aspects liés à la résilience. Les résultats montrent l'impact de ce facteur sur la réussiteléchec (RU) tant sur le plan quantitatif (descriptif et prédictif) comme sur le plan qualitatif. Dans tous les cas, la résilience assume un poids particulier face à la réussite, à l'épanouissement des universitaires et à la qualité de l'université (ici, des facultés). En conséquence, il faudrait la consolider à partir des institutions socialisatrices et des programmes de formation/emploi, notamment dans les pays moins développés où les difficultés à surmonter sont fréquentes.
\end{abstract}

Keywords / Mots-clés :

résilience, résistance, université, rallongement des études, pays émergents

\section{INTRODUCTION}

La mondialisation est accompagnée des nouvelles exigences, difficiles bien souvent à surmonter. Le manque suffisant de formation et de professionnalisation influe notamment: les gens n'ont pas développées des habilités/compétences nécessaires pour faire face aux obstacles. Cela conduit, bien souvent, à l'échec personnel, qui impacte sur les institutions, sur le contexte immédiat et, indirectement, sur le plan macro régional ou macro national.

Cette recherche est inscrite, précisément, dans ce cadre : la problématique générale de la Qualité des institutions (dans ce cas particulier : l'Université). Elle a été menée avec des sujets qui rallongent leurs études, comme réponse à une demande institutionnelle : il s'agit de la seule recherche promue par le Rectorat de l'Université Nationale de Cuyo, dont l'intérêt visait l'amélioration continue de la qualité de l'Université.

Une telle amélioration constitue un problème généralisé mis en évidence par les chiffres élevés de rallongement et d'abandon à l'Université. 
L'étude contribuerait à connaître en profondeur la réalité universitaire ainsi que les facteurs sousjacents à l'échec très élevé (mesuré pour le rallongement des études /niveau bas de réussite). Seul 11 $\%$ des étudiants globalement obtiendront leurs diplômes ; $70 \%$ abandonneront leurs études et le reste «trouvera la décision» de les rallonger. Nous-nous arrêterons ci-dessous sur ces statistiques. La problématique représente un coût matériel pour l'institution et humain pour les individus en termes de frustration. Parmi les réponses psychosociales des sujets face à l'échec apparaissent l'anomie, les vides, la dépression ou l'activisme, etc. (Aparicio, 2007a, 2007b; 2009a ; 2009 b). Les caractéristiques de la formation universitaire, basée plutôt sur le plan disciplinaire, serait à l'origine des chiffres ci-dessus cités. En effet, très fréquemment le système oublie la formation d'autres compétences nécessaires à la réussite universitaire et au monde du travail, comme le montre bien la littérature internationale (les compétences communicationnelles, les stratégies de coping, la résilience, parmi d'autres) (Aparicio, 2005, 2009, 2015 a et b), En ce qui concerne la résilience, axe de cette recherche, elle peut aider quelqu'un à développer des liens sociaux, des compétences relationnelles qui seront très utiles dans le monde universitaire, le monde du travail ainsi que dans la vie elle-même.

\section{CADRE THÉORIQUE}

La résilience peut être définie comme la capacité d'un individu à réagir et à surmonter les adversités. Cette capacité implique un ensemble de qualités favorisant un processus d'adaptation réussie et de transformation malgré les risques et/ou l'adversité elle-même.

Il importe de souligner que la capacité à résister aux pressions, à atteindre une vie saine même dans une ambiance adverse, tient, premièrement, à un ensemble de processus sociaux et intrapsychiques ; deuxièmement, en tant que capacité, la résilience concerne non seulement les individus, mais également les groupes (famille, communauté ou autres) : ils sont amenés à agir correctement et à réussir malgré les circonstances adverses, grâce à la croyance dans leur propre efficacité pour résoudre des problèmes et pour s'adapter au changement (Hernández, 1998 ; Puerta de Klinkert, 2002) ${ }^{1}$.

La résilience est donc une construction personnelle et institutionnelle. Alors, pourquoi travailler au sein d'institutions éducatives pour y analyser quels sont les aspects psychosociaux qui convergent vers le renforcement de l'homme et des institutions où il s'insère ?

Premièrement, notre approche est systémique sui generis. Ce qui nous intéresse c'est le sujet inséré dans un contexte. Un contexte qui favorise ou dilue les chances de devenir assez fort pour faire face aux obstacles et réussir au sens large du terme, pour franchir les barrières et atteindre certains buts, pour voir se cristalliser certains projets de vie.

Deuxièmement, il fallait comprendre les racines des problèmes très répandus chez les universitaires, où l'échec semble se multiplier. Une question s'impose : Une faible résilience chez les sujets et les « institutions », influe-t-elle au moment d'envisager les obstacles ? La communauté universitaire ellemême, avec son système privilégié de croyances, de valeurs et de pratiques, aurait-elle une influence sur les possibilités de réussir?

Sans connaître ni comprendre la situation, les chances de promouvoir des changements significatifs au sein de nos universités seraient très limitées, nous situant déjà sur le plan de l'intervention.

\section{Objectifs de la recherche :}

- Connaître les facteurs (de base, psychosociaux, structurels et pédagogique-institutionnels) associés à l'échec universitaire (ici, observé à travers le rallongement des études).

- Savoir quels aspects socioculturels et psychosociaux sont en rapport le plus fréquemment avec le rendement chez des sujets en retard aux effets : a) reconnaitre une population en risque ; b) fournir aux responsables les éléments afin d'en prendre une décision basé sur des données réelles, permettant la

\footnotetext{
${ }^{1}$ Pour la formulation des cadres théoriques pour ce sous projet il a fallu revisiter la théorie produite jusqu'à nos jours dans la matière. Présenter les apports de chaque auteur dépasse notre propos (cf. Aparicio, 2006, 2009, 2007b, 2016). Seulement nous faisons référence aux auteurs et notions-clés, en remarquant certains points en commun de ces auteurs et qui justifient qu'on les revisite.
} 
mise en marche de mesures préventives dans chaque faculté au niveau du plan d'études, du système d'évaluation et de promotion, des programmes de formation continue.

- Appréhender : a) sur un plan qualitatif, les raisons profondes qui sont à la base d'un phénomène très répandu au sein des universités argentines et dans le monde entier ; b) sur le plan quantitatif, connaitre quel est le poids de la résilience face à l'échec universitaire et quel est le niveau de résilience développé (trois échelles : étudiants, personnel, institution).

\section{Hypothèses générales :}

Deux hypothèses générales nous ont orientée: a) les facteurs psychosociaux -parmi lesquels se trouvent les motivations, le style attributionnel, le coping et la résilience dans notre modèleconsolidés par les premières instances socialisatrices et de formation (famille, pairs, école, les autres) et internalisés par les sujets, impacteraient sur des profils à plus forte réussite professionnelle car ils puissent faire face aux difficultés ; b) l'orientation précoce et les programmes de formation/prévention opèreraient à la manière de processus qui précèdent le comportement professionnel réussi.

\section{Hypothèse spécifique :}

Une haute résilience (RESIL) agit positivement sur la réussite universitaire (RU) et, indirectement, sur les possibilités d'insertion professionnelle dans un monde qui change vite et exige des nouvelles compétences sociales ${ }^{2}$.

Dans le cadre théorique, nous-nous arrêterons, un moment, sur les statistiques. Dans un second temps, nous ferons référence aux définitions de Résilience, en mettant l'accent sur la différence avec la notion de Résistance, et d'autres.

\subsection{Quelques chiffres}

En Argentine, les pourcentages d'abandon universitaire sont très hauts: entre $60 \%$ et $70 \%$ (Secrétariat des statistiques universitaires, SPU). Dans d'autres pays de la région, la moyenne d'abandon est proche au 55\%: Guatemala (82\%), Uruguay (72\%) y Bolivia (73\%), Brésil (59\%), Chile (54\%), Costa Rica (54\%) y Mexique (53\%) (Rojas, 2007). Ces chiffres nous montrent une situation qui affecte les sujets, les institutions eux-mêmes et aux Etats. González Lugo (2013) soutient que, par ans académique, l'Amérique latine et le Caraïbe, perdent entre 2 et 415 millions de dollars par pays, produit de l'abandon à l'université.

Dans d'autres pays développés, les taux d'abandon sont plus bas: Autriche, Espagne, Etats Unis et France, le taux oscille entre le 30\% et le 50\% ; en Allemagne (20\%-25\%), Suisse (7\%-30\%), Finlande (10\%) et les Pays Bas (20\%-30\%) (Vélez, 2005). Quant au rallongement des études, il existe très peu des recherches.

\subsection{De la résistance à la résilience}

\subsubsection{Quelques antécédents}

Analysons la définition de résilience et un corpus de concepts connexes, en montrant - nous l'avons dit - les aspects qui la définissent. Cela nous donnera l'occasion de la présenter comme une notion dynamique et un processus. Nous ferons référence aussi aux niveaux de résilience et aux derniers aspects qui la définissent, ainsi qu'à l'approche épistémologique qui est à la base et que, depuis l'évolution connue, nous partageons.

Le concept de résilience n'est pas nouveau. D'abord a été utilisé en métallurgie et ingénierie pour décrire la capacité de certains matériaux de récupérer la forme originale d'après être soumis à une

\footnotetext{
${ }^{2}$ Même si trouver un diplôme universitaire aujourd'hui n'assure pas l'insertion ni la permanence professionnelle, les recherches et les statistiques internationales montrent bien qu'un diplômé a plus de possibilités d'épanouissement et de réalisation professionnelle possédant un diplôme qu'un non diplômé (ère de la connaissance et des certifications).
} 
pression qui déforme (Real Academia de la Lengua; Enciclopedia Salvat de la Ciencia y de la Tecnología, 1964).

Historiquement, on lit en Dyer et al. (1996) (t.n.):«La résilience est un mot utilisé pour décrire la qualité flexible ou élastique d'une substance Le Oxford English Dictionary (1933) définit la résilience comme «1. Le (ou un) acte de récuperation». L'American Heritage Dictionary (1994) la définit comme « la capacité de se récupérer d'une maladie, changement ou fait malheureux » (p. 1534).

En psychologie positive la notion entre vers les années 60 et, plus encore, pendant les années 70 et 80 (Seligman \& Csikszentmihalyi, 2000) ${ }^{3}$.

Les études longitudinales menées par Werner \& Smith (1982) avec des cohortes multiraciales, exposées aux risques à Hawaï, sont décisives pour établir une différence entre Résilience et Résistance (Hardiness), Apres être utilisés indistinctement Invulnérable et Invincible par Anthony $(1974 ; 1987)$ dans la littérature psychiatrique précoce ${ }^{4}$.

C'est comme ça que les concepts d'invulnérabilité et invincibilité (Anthony, 1974; Werner \& Smith, 1982) ont perdu sa force en faveur de la résilience, une notion dynamique, en raison de se traiter des qualités « fixes » et « statiques ».

Les auteurs découvrent, donc, des facteurs protecteurs : auto-estime, indépendance, liens, initiative, pensé critique; facteurs qu'ils considèrent les piliers de la résilience. Ils soulignent la différence entre la physique et la psychologie. En physique la résilience renvoie à la capacité de retourner vers un état antérieur aux changements supportés par les forces externes; en revanche, en psychologie, ce concept est plus large. Il ne s'agit pas de retourner à un état antérieur. L'appareil psychique se reconstitue en adoptant une organisation plus efficace plus faire face au futur, après l'impact du trauma qui a provoqué la douleur émotionnelle (Lorenzo, 2010)5.

\subsubsection{Sur quelques caractéristiques de la résilience}

\subsubsection{Une notion dynamique}

On peut considérer le «père fondateur» Michael Rutter (1985; Rutter, MacDonald, Le Couteur, Harrington, Bolton \& Bailey, 1991). A partir d'une perspective psycho-biologique, il conçoit les facteurs protecteurs non comme contraires aux facteurs de risque. Il s'agit d'une dynamique qui permet sortir au sujet fortifié face à l'adversité, en chaque situation et en respectant les caractéristiques personnelles (Rutter, 1991). De sa part, Edith Grotberg (2003) définit la Résilience comme l'interaction des facteurs placés a 3 niveaux: le support social (j'ai); les habilités (je peux) et la forteresse interne (je suis et j'ai ici). L'essentiel est l'interaction entre le sujet et son contexte, ayant les personnes toujours un rôle actif face aux évènements.

Kotliarenco, Cáceres \& Fontecilla (1997) ont décrit l'évolution de la notion de résilience en 3 moments. Le premier est constitué pour l'acceptation d'une "adaptation positive" ayant les sujets « invulnérables », « résistantes », c'est-à-dire, pouvant « résister » l'adversité (Wolin \& Wolin, 1993; cit. par Henderson, 2003).

\footnotetext{
${ }^{3}$ En langue espagnole, le concept est introduit plus tard, vers 2001-2005 (Cuervo Rodríguez, Yanguma \& Arroyave, 2011). Les chercheurs manifestent un intérêt particulier pour découvrir et analyser les facteurs qui sont à la base de l'adaptation positive (Santos, Kotliarenco, Néstor Suárez Ojeda, Infante, Grotberg, 2008).

${ }^{4}$ On lit en Dyer \& and Minton McGuinness: "Werner \& Smith (1982) on fait une recherche longitudinal avec la cohorte de naissance de 1955 de 698 enfants dans l'ile de Kauai, Hawai. Plusieurs de ces familles habitaient dans la pauvreté, et plusieurs membres avaient des dis capacités mentales et phtisiques. Les chercheurs se sont centrés sur la combinaison des facteurs biologiques, sociaux et psychologiques qui étaient considérés prédictifs de vulnérabilité et d'invulnérabilité à des problèmes graves de coping. Ils ont observé que les jeunes "invulnérables", qui avaient subi moins de séparations prolongées de leurs mères dans le premier an de vie, étaient des bébés actifs et socialement réceptifs, bien souvent ils étaient des fils «primogenites » et ils avaient accompli les normes de manière consistante » (cf. aussi, Werner, 1989 ; 1995).

${ }^{5}$ Kotliarenco, Cáceres \& Fontecilla (1997) renvoient à l'origine latin du mot : résilience vient de « resilio », c'est-à-dire, aller en arrière en faisant un saut qualitatif.
} 
L'invulnérabilité est, donc, la première notion. Elle renvoie aux personnes qui apparaissent comme très fortes, ne cédant pas aux pressions du stress et de l'adversité.

Cependant - comme nous venons de signaler plus dessus - la notion a été considérée erronée depuis un temps parce que la résistance à le stress est relative, n'est pas stable dans le temps et varie d'accord à l'étape du développement des sujets et aux circonstances. Les racines de la résilience, de plus, proviennent tant de l'ambiance comme de la constitution du sujet (Rutter, 1985; Klotiarenco et al. 1997).

Le deuxième moment est constitué par le remplacement du concept d'invulnérabilité par celui de résilience : la résilience peut se former/consolider, en tant que la invulnérabilité est considéré un trait intrinsèque du sujet (Rutter, 1991).

Barnard (1999) et Manciaux, Vanistendael, Lecomte \& Cyrulnik (2001, cit. par Manciaux, 2003) partagent la même perspective: la résilience est un processus dynamique; les influences du contexte (familial, social, culturel) et les forces de l'individu (facteurs internes) interagissent réciproquement permettant dépasser le risque. Cela permet à la personne de s'adapter à l'adversité (González-Arratia, 2007)

\subsubsection{Un «processus »}

De plus, il s'agit d'un processus existant dans les personnes normales mais non comme une capacité innée (Grotberg, 1995 ; 2006 ; 2002, cit. par Melillo \& Suarez Ojeda ; Trujillo García, 2011). Même si elle suppose une réponse individuelle, elle est conditionné tant pour des facteurs individuels comme ceux de l'ambiance. En effet, il faut la former, la construire dans un processus interactif sujet-contexte (Rutter, 1993 ; 1992). La notion inclue tant les relations sociales comme les processus intrapsychiques (motivation, représentations, ajustement,...). Le tout permet au sujet d'avoir une vie normale dans un contexte pathogène.

Trujillo García (2011) aussi considère la résilience comme un processus et pas comme une capacité innée. Face à l'adversité, on peut chercher l'amélioration personnelle et collective.

La nature de la résilience est donc dynamique et elle est construite par la personne en fonction de soimême et du contexte. Il n'y a pas de déterminisme génétique ou du contexte sur la personne. Cela suppose une nouvelle approche épistémologique de l'être humain, en tant qu'elle met l'accent sur le potentiel humain, est spécifique de chaque culture et convoque la responsabilité collective. A partir du moment qu'on la considère un processus, on peut penser qui peut se développer. De plus, étant une capacité qui se développe et se reconstruit dans l'histoire du sujet, sa relation avec les diverses étapes de la vie assume une particulière relevance.

En conséquence, il est intéressant de noter que quand il faut faire face aux changements et aux crises de la vie, comme est le passage par l'université, est ou la promotion de la résilience marque la différence de la prédisposition au développement des potentialités humaines ou de son étanchement (Saavedra Guajardo \& Villalta Paucar, 2008).

\subsubsection{Sur des niveaux de la résilience}

Arrêtons-nous un moment sur les niveaux de la résilience car dans nos résultats nous ferons référence à ceux-ci.

Lorenzo (2010) soutient qui peut se distinguer 3 niveaux : individuel, groupal et social. Chaque sujet développe sa capacité pour dépasser les conflits de manière individuelle, mais quand un évènement affecte au groupe ou à la société dans laquelle il habite, la réaction est produite en bloque. Le groupe et/ou la société réagissent comme une totalité structurée dans laquelle les réponses individuelles exercent son influence sur les autres et sur le tout. Il ne s'agit pas d'une agrégation d'individualités: c'est qui fait possible ce phénomène est la cohésion. Il y a de cas ou les sujets développent cette capacité mais ils n'aboutissent pas à développer cette capacité à niveau groupal ou social.

De même, l'auteur soutient que la résilience se développe de diverses manières en chaque individu. Un exemple de niveau bas : le sujet résoudre une situation mais les conséquences à niveau émotionnel 
sont autant importante qui l'empêchent continuer. Alors, sa capacité de réagir est bonne mais sa résilience est basse.

Exemple d'un niveau haut: le sujet résoudre la situation et va plus delà de l'impact émotionnel. De cette manière, il mène des mécanismes nécessaires pour continuer avec sa vie, en le donnant un nouveau sens et des possibilités pour la construction de nouveaux projets.

Dans le pire des cas, le sujet ne fait face aux conflits stressants et n'aboutit pas à résoudre la situation. Ici on peut parler du manque de résilience ou de personnes non résilientes.

\subsubsection{Types de résilience}

Concernant encore les divers types de définition sur la résilience, García-Vesga \& Domínguez-de la Ossa (2013) présentent une division: 1) celles qui mettent l'accent sur l'adaptabilité; 2) celles qu'incluent le concept de capacité ou d'habileté; 3) celles qui se focalisent sur l'interaction des facteurs internes et externes ; 4) celles qui définissent la résilience comme une adaptation et processus. Toutes les définitions accentuent le rôle actif du sujet.

Vanistendael (1994, 1995 ; 2001 ; Vanistendael \& Lecomte, 2002) soutient que toutes les définitions, au moins, incluent deux aspects: 1) la résistance ou la capacité de conserver l'intégralité face aux "coups"; b) la capacité de construire ou de se réaliser positivement en dépit des difficultés.

Cependant, n'existe pas de consensus sur la définition.

Avant de finir cet item, faisons référence à d'autres notions, très liées et/ou confondues avec la résilience.

\subsubsection{D’autres notions liées à la résilience}

Il y a des notions associées.

\section{-Compétence}

Elle est définie comme habilité sociale ou réussite dans une activité (Becoña, 2006). La compétence suppose l'effectivité en acte (exécution dans un domaine). Masten (1999; 2001) soutient que "compétence" et "résilience" sont deux constructions très liées entre soi, constituant une partie d'une construction plus général d'adaptation. Luthar (1993; 2000a and 200b ; 2006, souligne la différence entre les deux notions: la compétence ne suppose pas de risque.

-Adaptation positive: S'adapter suppose s'ajuster, c'est-à-dire, comporte des changements internes et dans le type des relations (Lorenzo, 2010). Mais seulement nous parlons d'adaptation résiliente s'il existe de l'adaptation positive.

-Forteresse: Il existe une différence entre forteresse et résilience. La dernière seulement tient en compte les comportements adaptatifs et constructifs dans la lutte pour réussir la situation. Pour cela, une personne qui ne tombe pas dans une infraction sociale, peut être considérée comme un sujet fort mais pas comme résilient (Novella, 2002).

-Coping: Lazarus \& Folkman $(1980$; 1986) définissent le coping comme les efforts cognitifs et comportementaux pour améliorer les demandes spécifiques externes y/o internes qui sont évaluées débordant les ressources de l'individu.

Mais ils existent des différences: 1) la résilience suppose toujours un comportement adaptatif ; 2) de plus, pour qu'il existe de la résilience, il faut qu'il existe une situation adverse et/ou stressante. En revanche, dans le coping, la réponse peut être adaptée ou non face aux événements quotidiens ; 3) la résilience suppose des stratégies d'ajustement efficaces; c'est-à-dire, des efforts pour maintenir l'équilibre interne ou externe à travers des activités concernant la pensée et l'action. Les individus doivent s'adapter aux évènements stressants et pour cela, ils évaluent la situation en appliquant des stratégies d'ajustement (Manciaux et al, 2003). 


\subsubsection{Un bilan :}

\section{* Quant à l'approche}

Dans notre cadre, il est important de remarquer que la résilience a introduit une perspective ou approche optimiste, pas unilatérale et plutôt systémique et complexe, tant dans la psychopathologie comme dans l'éducation. En effet, on peut confier plus dans les forteresses des personnes et dans ses possibilités de réaliser des changements positifs aidés par l'ambiance. L'évolution montre le passage d'une réflexion qui va du plan personnel vers le plan relationnel, constituant une théorie écologique de la résilience (Bronfenbrenner, 1981, 1987) ; une théorie qui s'insère dans la théorie de l'auteur, intitulé The Three Dimensional Spiral of Sens (2015). L'évolution montre, donc, le passage vers une perspective de la complexité (Aparicio, 2007 a et b) ${ }^{6}$.

Selon Grotberg (2005), on peut distinguer huit (8) nouveaux aspects à partir du concept de résilience. 1) La résilience est liée au développement humain incluant des différences d'âge et de genre; 2) pour consolider la résilience il faut faire appel à différentes stratégies; 3) la résilience n'est pas liée au niveau socio-économique; 4) la résilience est différente aux facteurs de risque et aux facteurs de protection ; 5) la résilience peut être mesurée et elle fait part de la santé mentale et de la qualité de vie ; 6) les différences culturelles diminuent quand les adultes sont capables de valoriser des nouvelles idées visant le développement humain ; 7) la prévention et la promotion sont des aspects liés à la résilience ; 8) la résilience est un processus : il y a des facteurs de résilience, des comportements résilients et des résultats résilients.

\section{* Quant aux apports de la résilience sur le plan universitaire}

Traditionnellement, les institutions éducatives se sont occupées de détecter des erreurs en lieu d'identifier les forteresses. Dans ce cadre, la résilience joue un rôle essentiel: à travers son développement on peut favoriser le développement des compétences sociales, académiques et personnelles qui aident aux étudiants à dépasser les difficultés et réussir (Rutter, 1987). Dans cette perspective, le plus important test de mettre l'accent sur l'acquisition et développement des compétences, capacités et des forteresses et non pas sur les faiblesses. L'éducation assume un rôle central visant la consolidation de la résilience dans des enfants, des adolescents et des universitaires eux-mêmes, pour les aider à faire face aux difficultés académiques et, après, dans le monde su travail (surtout si les sujets appartiennent aux contextes défavorisés (Melillo, Rubbo \& Morato, 2004 ; Coronado-Hijón, 2017).

Pulgar Suaso (2010) soutient qu'un étudiant résilient s'engage, est responsable et tout cela influe sur la réussite, la baisse du rallongement des études, l'insertion professionnelle, la productivité, parmi d'autres aspects (Acevedo \& Mondragón, 2005), montre bien que un contexte institutionnel qui favorise la résilience parmi leurs étudiants; agit sur le plan de la prévention de crises. Les enseignants peuvent aussi, dans ce cadre, identifier les étudiants en situation de risque pour les aider à exploiter leurs potentialités pour faire face aux obstacles (González \& Valdez, 2006, cit. par Pulgar Suaso, 2010).

En dépit de l'importance de la résilience, elle a été très peu étudiée dans des institutions éducatives et, notamment, dans l'éducation supérieure. Henderson \& Milstein (2003) soutiennent que la plupart de recherches concernant la résilience ont été faites sur populations des enfants et des adolescents.

De plus, selon Haz \& Castillo (2003), les études, en général, n'approfondissent pas sur les « conditions »/ « qualités » personnelles. Cela justifie notre recherche.

En général, on peut dire que la résilience est une notion d'un potentiel énorme pour tous les professionnels de l'éducation et de la santé (Coronado-Hijón \& Paneque, 2015).

Manciaux et al. (2003) propose une définition pragmatique qui a leurs racines dans les réalités éducatives quotidiennes afin de développer les habilités 'pour sortir de l'adversité, s'adapter et accéder a une vie productive et avec du sens.

\footnotetext{
${ }^{6}$ On peut consulter, aussi, d'autres modèles théoriques de résilience, d'abandon et de permanence (Coronado Hijón, 2017 ; Dubs, R. (2005).
} 
Résister est créer les conditions. Le processus de création est positif, mais la résilience es le moyen pour aboutir à l'œuvre personnelle.

On peut dire qu'il est d'intérêt pour les individus et pour les organisations/institutions elles-mêmes, travailler sur le plan de l'intervention à partir de la psychologie éducative et sociale à l'Université avec des programmes de leadership, motivation, participation et de responsabilité. De même, avec des programmes sur les habitudes d'étude, de travail sur les projets de vie en jetant lumière sur les buts pour qu'ils deviennent plus clairs, etc. Et tous ces facteurs sont des variables/dimensions de notre modèle dans cette recherche.

Autrement dit, le travail avec les facteurs personnels des étudiants depuis le modèle de la résilience qui se centre sur les caractéristiques positives des sujets-, peut servir pour faire face aux situations de risque académique ou d'autres et permettre à l'individu de récupérer un certain équilibre (homéostasies). Pour cela est très important d'identifier les facteurs résilients qui se relient significativement avec la réussite des étudiantes.

Henderson \& Milstein (2003) ont analysé la résilience dans des milieux éducatifs formels. Ils détectent d'abord, 6 étapes pour fortifier la résilience, à travers l'intervention de la famille, l'école et la communauté elle-même.

Les auteurs considèrent qui peut être conçue comme une stratégie pour la récupération de l'homéostasie qui permet à l'individu chercher l'équilibre qui donne comme résultat la tranquillité. Ils élaborent un modèle donc l'objectif est de répandre la résilience dans des contextes formels. Il y aurait six (6) étapes pour fortifier la résilience à travers la famille, l'école et la communauté: 1 . Donner de l'affection et de l'appui inconditionnels, comme base de la réussite académique. 2. Etablir et transmette des attentes élevés et réalistes, tous les étudiants peuvent apprendre. 3. Donner des opportunités de participation significatives dans la résolution de problèmes. 4. Enrichir les liens avec la communauté éducative en cherchant la connexion famille-communauté 5. Former au personnel sur des stratégies et des politiques de la salle de classe, et donner de participation à la communauté éducative. 6. Apprendre des habilités pour la vie, la coopération, les habilités communicationnelles, pour prendre de décisions. Trois de ceux-ci ont pour objectif de mitiger les facteurs de risque qui proviennent de l'ambiance: enrichir les liens pro-sociaux, fixer des limites clairs et firmes dans l'activité éducative et apprendre des habilités pour la vie. Les pas ou «étapes » 4 au 6 sont ceux qui fortifient la résilience: donner de l'affection de l'appui, établir des attentes hautes, réalistes mais qui puissent se voir cristallisés et, en dernier terme, donner des opportunités de participation significative.

Des 6 pas ou «étapes », le premier est le plus important et permanent: donner de l'affection, de l'amour: il parait, en effet, qu'il peu possible de surmonter les difficultés et d'accroitre dans le sens humain sans avoir de l'amour des autres.

Les résultats de ces 6 étapes, engendrent des personnes optimistes, responsables, avec une haute estime de soi et d'auto-efficacité. De plus, les auteurs que nous suivons dans l'essentiel dans cette recherche, considèrent que l'implantation de ces bonnes pratiques de consolidation de la résilience académique - qui peuvent améliorer l'apprentissage et développer des compétences, des talents et défendre face aux risques - doit être vertébrée sur 3 niveaux de l'Administration éducative : les politiques éducatives, le centre éducatif et, finalement, le corps enseignant.

La résilience, donc, augmente les probabilités de réussite éducative, plus delà de la vulnérabilité, qui vient des expériences et des conditions du contexte (Wang, Haertl \& Walberg, 1990, 1994).

Rirkin \& Hoopman (1991) définissent la résilience dans le champ éducatif comme « la capacité de se récupérer et de s'adapter avec réussite face à l'adversité et de développer la compétence sociale, académique et vocationnelle, malgré être exposé à un stress haut ou, simplement, aux tensions inhérentes au monde d'aujourd'hui » (Henderson \& Milstein, $2003: 26$ )

Il faut dire que plusieurs recherches montrent bien la force la résilience face à la réussite (Erberber et al., 2015), émergeant avec le poids le plus important. Dans cette recherche - nous l'observerons - la résilience a aussi la plus grande importance parmi les facteurs d'échec (Aparicio, 2009 a ; 2009 b ; 2016, 2018). 
Le Rapport sur l'enquête du PNUD 2004; dit que les sujets qui manifestent moins de confiance en soimêmes, ont moins de possibilités d'usage des réseau institutionnels, sociaux, familiales et un mineur control sur son futur, sur son «destin »: De plus, eux-mêmes déclarent se sentir exclus et connaitre moins d'opportunités.

Goleman (1996) souligne que l'accent mis sur des modèles cognitifs-technologiques et pas sur les compétences sociales dans l'enseignement supérieur, favorise l'émergence parmi les étudiants de la peur, de l'insécurité, de l'instabilité, des frustrations face à l'adversité, qui peuvent induire désajustements sociaux et le manque de contrôle de soi.

Le Rapport Delors (UNESCO, 1996), a signalé les quatre (4) piliers d'une politique éducative de qualité. Celle-ci inclue: le savoir, le savoir-faire, le savoir-vivre avec les autres et l'apprendre à être. Les deux premiers aspects se correspondant avec les modèles traditionnels: il s'agit de mesurer pour justifier les résultats. Les deux derniers deviennent de plus en plus importants dans le cadre du monde actuel et de la mondialisation pour favoriser l'intégration sociale et la construction de la citoyenneté. Les programmes concernant la résilience entrent dans les deux derniers aspects.

\subsection{D'autres notions liées à la résilience}

Il y a des notions associées. Arrêtons-nous un moment sur les plus confondues.

\subsubsection{Compétence}

Elle est définie comme habilité sociale ou réussite dans une activité (Becoña, 2006). La compétence suppose l'effectivité en acte (exécution dans un domaine). Masten (2001) soutient que "compétence" et "résilience" sont deux constructions très liées entre soi, constituant une partie d'une construction plus générale d'adaptation. Luthar (2006, cit. par Becoña, 2006), souligne la différence entre les deux notions: la compétence ne suppose pas de risque ${ }^{7}$.

\subsubsection{Adaptation positive:}

S'adapter suppose s'ajuster, c'est-à-dire, comporte des changements internes et dans le type des relations (Lorenzo, 2010). Mais seulement nous parlons d'adaptation résiliente s'il existe de l'adaptation positive.

\subsubsection{Forteresse:}

Il existe une différence entre forteresse et résilience. La dernière seulement tient en compte les comportements adaptatifs et constructifs dans la lutte pour réussir la situation. Pour cela, une personne qui tombe dans une infraction sociale, peut être considérée comme un sujet fort mais pas comme résilient (Novella, 2002).

\subsubsection{Coping:}

Lazarus \& Folkman (1986) définissent le coping comme les efforts cognitifs et comportementaux pour améliorer les demandes spécifiques externes y/o internes qui sont évaluées débordant les ressources de l'individu.

Mais ils existent des différences: a) la résilience suppose toujours un comportement adaptatif ; b) de plus, pour qu'il existe de la résilience, il faut qu'il existe une situation adverse et/ou stressante. En revanche, dans le coping, la réponse peut être adaptée ou non face aux événements quotidiens ; c) la résilience suppose des stratégies d'ajustement efficaces; c'est-à-dire, des efforts pour maintenir l'équilibre interne ou externe à travers des activités concernant la pensée et l'action. Les individus doivent s'adapter aux évènements stressants et pour cela, ils évaluent la situation en appliquant des stratégies d'ajustement (Manciaux et al, 2003).

\footnotetext{
${ }^{7}$ Voir aussi Luthar, 1993 ; 2000a, 2000 b,
} 


\section{MÉTHODOLOGIE}

Elle a été quantitative et qualitative.

\subsection{L'échantillon :}

Il a été était constitué par de 229 sujets de six facultés de l'Université Nationale de Cuyo (1985-2004), que nous avons interviewés à domicile. Il s'agit des sujets qui, inscrits à l'Université depuis 1985 et réinscrits pendant l'année scolaire universitaire 2004, s'absentent des cours : ce sont des étudiants «fantômes ». Ils sont inscrits dans des formations relevant de six facultés : Philosophie et lettres (Sciences de l'éducation), Sciences économiques (Comptabilité, Gestion) ; Sciences politiques et sociales (Communication sociale), Droit, Médicine et Génie (Civil, Industriel et en Pétrole). Sur un total de 1880 sujets identifiés d'après leurs dossiers, le nombre effectif des sujets enquêtés (que nous avons pu localiser) a été de $\mathrm{N}=229^{8}$.

\subsection{Les techniques :}

Nous avons utilisé une enquête semi-structurée et des tests spécifiques pour mesurer les variables psychosociales en rapport au avec le niveau de réussite (RU): les Stratégies pour «faire face » aux difficultés (Frydenberg \& Lewis, 1996), les styles attributionnels (Seligman, 1991), les facteurs motivationnels (Montero \& Alonso Tapia, 1992) et la Résilience (Henderson \& Milstein, 2003). Dans cet article nous ne traitons que la Résilience et son importance pour faire face aux difficultés au sein des institutions universitaires. .

Pour ce qui est de la Résilience, le Questionnaire de Henderson et Milstein est composé de 3 échelles - Étudiant (RESIALUM), Personnel - administratif et enseignant - (RESIPERS) et Institutionnel (RESIFACU) - et 6 sous-échelles qui peuvent être groupées en 2 sous-dimensions. Les aspects y évalués sont: I. Mitiger le risque: 1 . Enrichir les liens pro-sociaux ; 2 . Fixer des limites claires et fermées (Élaborer et mettre en place des politiques et des procédures scolaires cohérentes et expliciter les attentes en termes de comportement. Exprimer des règles par écrit et les transmettre de façon claire); 3. Enseigner des compétences pour la vie (coopération, résolution de conflits, compétences communicationnelles, habileté pour résoudre des problèmes et prendre des décisions, gestion saine du stress). II. Construire de la Résilience: 1. Donner de l'affection et du soutien - c'est l'élément déterminant crucial ; 2. Transmettre des attentes élevées et réalistes, attendre le mieux (éviter le recours à des étiquettes et la notion de plafond du développement) ; 3 . Donner des chances de participation significative (accorder aux étudiants et aux responsables institutionnels de la responsabilité dans la résolution des problèmes, la prise de décisions, la définition d'objectifs/ les buts et l'aide aux autres).

Pour ce qui est de la Réussite universitaire (RU), elle a été interprétée dans un sens large et un sens strict du terme.

Rendement au sens large du terme. Il comporte les catégories suivantes.

-Succès : fin de la carrière - obtention du diplôme -.

-Retard : fin de la carrière dans une période de temps plus longue que prévue officiellement

(Diplômés quelques années plus tard).

-Echec : abandon de certains cours.

Rendement au sens strict: Conçu d'après les items suivants fournis par la Direction de Statistiques de l'UNCuyo'.

-Années de permanence dans la carrière (2005- cohorte)

-Durée de la carrière d'après le Programme d'Etudes correspondant à l'étudiant (ANPLAN)

-Matières non-réussies (MATPLAN-REUSSI)

\footnotetext{
${ }^{8}$ La recherche a continué après comme projet institutionnel de notre Université.

${ }^{9}$ Pour les formules appliquées, consulter Aparicio 2009 a.
} 
-Cours correspondants à la carrière d'après le Programme d'Etudes auquel appartient l'étudiant (MATPLAN)

\section{-Quantité d'échecs (APLAZOS)}

-Matières réussies (MATPLAN)

Toute l'information (qualitative et quantitative) a été finalement triangulée. Les outils existants à ce jour saisissent le phénomène, en général, de manière unilatérale, mettant l'accent sur le sujet (la résilience, le coping). Or la résilience concerne aussi les institutions. Il s'agit d'une condition, «qualité » ou compétence qui n'est pas innée : c'est aux institutions/organisations de les réaffirmer, voire même de les former.

\section{RÉSULTATS}

Ils montrent le rôle joué par la résilience sur la réussite et le rallongement des études.

Passons maintenant en revue quelques résultats. Rappelons que le facteur Résilience a été mesuré au moyen de trois échelles: Etudiant (RESIALUM), Personnel - administratif et enseignant (RESIPERS) et Institutionnel (RESIFACU).

Les variables ont une ponctuation inaperçue de sorte que le n. 1 indique : On est bien, 2. On a avancé, 3. On commence, 4. On n'a rien fait. La ponctuation élevée ou maximale de 48 démontre que l'étudiant évalue la formation de résilience comme nulle ou inexistante. Par contre la valeur minimale du test pour les échelles étudiant/personnel et institution est de 12, ce qui démontre que l'interviewé a évalué la formation de résilience de la part de la faculté comme quelque chose de positif. Bref, on la conçoit comme formatrice de résilience.

Autrement dit, pour les trois échelles - Étudiant, Personnel et Institutionnel - la moyenne s'est concentrée à la catégorie 3, ce qui indique que la formation de Résilience «est à ses débuts ». Ceci signifie qu'en termes généraux, dans toutes les facultés, l'évaluation des institutions comme formatrices de Résilience chez l'étudiant, le Personnel et dans l'Institution elle-même est assez basse. Par ailleurs, chaque institution offre un profil propre, certaines d'entre elles apparaissant comme génératrices de Résilience chez les étudiants, le personnel et/ou l'institution elle-même ${ }^{10}$.

Nous ne nous arrêtons pas sur le traitement détaillé en renvoyant à Aparicio $(2016 ; 2018)$.

Les résultants montrent qu'il y aurait des espaces générateurs de résilience (lire facultés/filières dans notre recherche) qui favoriseraient son émergence, c'est-à-dire, des espaces qui causent/favorisent : a) la génération de réseaux sociaux informels assurant une acceptation inconditionnelle; b) la capacité à trouver un sens transcendant aux événements de la vie ; c) le développement de capacités à nouer des relations solides, respectueuses et gratifiantes, à respecter des normes et des limites de manière consciente; d) la capacité à résoudre des problèmes au moyen de la réflexion et l'analyse ; e) le développement de l'auto-estime à partir d'une vision réaliste de ses propres potentiel et limites.

Esquissons à présent d'autres résultats quantitatifs qui concernent des aspects qui influent sur la réussite (pour l'analyse en détail on renvoi à Aparicio, 2016a, b, c ; 2018).

Analyse bivariée : Quant aux facteurs pour «faire-face » aux obstacles de notre modèle - Résilience et Coping -, les résultats démontrent que les échelles de Résilience les plus significatives parmi celles mesurant la formation de Résilience ont été le Lien pro social (RESIVINC), les limites claires (RESILI) et les Attentes élevées (RESIEXPE). Plus précisément, lorsque le Lien pro social augmente, les Rendements universitaires diminuent (RU) ; en revanche lorsqu'il y a des Limites claires et des Attentes élevées, les Rendements universitaires (RU) augmentent.

On y remarque donc l'incidence positive des attentes par rapport à l'apprentissage et à la connaissance des limites ; à l'inverse, joue le lien pro social très haut, qui conduirait à négliger les études. Sur le

\footnotetext{
${ }^{10}$ Nous attarder sur le profil typique de chaque faculté impliquerait de trop rallonger cette présentation. Voir Aparicio, 2007a, tome 1 .
} 
plan des exigences, l'expérience dans différentes facultés (ayant changé leurs plans d'études pour d'autres, plus exigeants et avec plus des limites), c'est que, à plus grandes limites, meilleurs sont les résultats en termes de rendement.

Analyse multi variée : Arrêtons-nous un moment sur le modèle de la réussite universitaire (RU). Le modèle inclut des variables de base, psychosociales, psychopédagogiques, pédagogique-institutionnels et structurelles.

Parmi les variables Psychosociales, celle qui émerge avec le plus grande force est la Résilience. Deux sous-échelles y sont associées donc à Réussite universitaire (RU): l'existence des liens et les opportunités offertes aux sujets (RESIVINC et RESIOPOR). La première contribue de façon négative, c'est-à-dire, plus les étudiants se consacrent à la vie sociale, moins de Rendement atteignent-ils. La deuxième contribue de façon positive : autrement dit, plus ont -ils des chances de progrès, meilleur sera leur Rendement Universitaire.

RESIVINC: la probabilité est inférieure à 5\% (0.03289). Le coefficient en est de -0.003520 , ce qui suppose un rapport négatif avec RU. En d'autres mots, quand ces liens sont exagérés et la sociabilité trop marquée, l'étude perd de son importance et les temps des études se rallongent.

RESIOPOR: la probabilité est inférieure au 5\% (0.00057), ce qui indique une association dans le modèle multi varié plus élevée que RESIVINC. Le coefficient en est de 0.004104 , ce qui indique un rapport positif avec RU. Cela revient à dire que lorsqu'il existe des opportunités de développement, les rendements académiques s'améliorent et le rallongement diminue.

\section{DISCUSSION}

Ces résultats constituent dans ce cadre un apport significatif : ils mettent en évidence la nécessité de développer des milieux de sources de résilience au sein des organisations de formation visant à la consolidation de la résilience (Aparicio, 2005, 2007a, 2007b, 2008, 2009a, b et c;2015 a ; 2015 b; $2016 \mathrm{a} ; 2016 \mathrm{~b} ; 2016 \mathrm{c} ; 2018)$. Elle contribuera aux possibilités de réalisation et d'une vie saine, même si les ambiances aujourd'hui et, de plus en plus, présentent des exigences qui paraissent déborder les capacités des sujets pour faire face.

De plus, étant donnée la richesse de la notion de résilience, que va plus delà de la simple résistance liée dans un sens large à d'autres facteurs qui aident les personnes à réussir - nous a permis de revisiter les axes de diverses micro-théories complémentaires (cf. Aparicio, 2005, 2009a, b et c, 2016) ${ }^{11}$. Ces variables/facteurs, sous-jacents à la réussite/échec objective et subjective des sujets -

buts, attentes réalistes, capacité de création de liens solides, respectueux, gratifiants ; communication, habileté pour résoudre des problèmes et prendre des décisions ; effort, limites -contribuent à faire face aux obstacles dans une société ou, bien souvent, la violence se répand et les limites se perdent.

Finalement, l'influence du contexte apparait nettement, surtout dans l'analyse qualitative. N'est pas notre objectif nous arrêter sur ce plan ${ }^{12}$. Disons, tout simplement, que les dimensions les plus importants et qui présentent plus de saillance pour les sujets qui ont rallongé leurs études, sont celles qui concernent l'interaction enseignant/étudiant; étudiant/étudiant et étudiant/organisation de la Faculté. Les liens solides, le respect, l'écoute, l'appui, la communication, parmi d'autres aspects, émergent comme facteurs fondamentaux à l'heure de continuer avec les études ou de prendre la décision de les abandonner ou de les rallonger.

Sujet et contexte, tous les deux en interaction constante dans un mouvement enrichissant ou appauvrissant pour le sujet et pour le contexte. Ceci tiendra au type d'interaction qui s'établit, de la capacité des institutions à former des capacités pour faire face aux obstacles, même s'il existe des

\footnotetext{
11 Théorie de l'auto efficacité (Bandura, 1977, 1982, 1987), du Need-ach (Besoins d'accomplissement, Mc Clelland, 1960), des Attentes/Valences (Feather \& Davenport, 1981) des Styles attributifs (Optimisme, Seligman, 1991, 2000) ; du Coping (Lazarus \& Folkman, 1986) et des Stratégies pour faire face aux obstacles (Frydenberg \& Lewis, 1996).

${ }^{12}$ Cela serait l'objet d'un autre article pour les détails qui suppose la présentation.
} 
limites contextuelles. D'une part, la socialisation émerge au cœur des résultats dans la ligne de la réalisation des gens. D'autre part, la psychologie sociale et la formation surgissent indissociablement liées dans cette contribution au développement humain et social. Le tout s'insère dans la théorie de l'auteure, «L'spirale trois dimensionnel $\mathrm{du}$ sens»: sujet/context/sujet ou plan macro/méso/micro/macro, avec les «allers-retours » ou des mouvements récursifs et des effets positifs et négatifs, permettant ou non une meilleure réalisation des hommes et des organisations elles-mêmes.

\section{CONCLUSION}

La compréhension de l'influence exercée par certains facteurs psychosociaux à la lumière des théories concernant la réussite pourrait favoriser, en effet, l'implantation des stratégies pour faciliter la réalisation des gens et la transformation des pratiques, notamment dans les pays moins développés ou émergents, où les difficultés à surmonter sont fréquentes.

\section{RÉFÉRENCES}

Anthony, E.J. (1974). «The Syndrome of the Psychologically Invulnerable Child. In E.J. Anthony \& C. Koupernik (Eds.), The Child in his Family: Children at Psychiatricrisk (pp. 3-10). Nueva York: Wiley.

Anthony, E.J. (1987). Risk, Vulnerability, and Resilience. In E.J. Anthony \& B.J. Cohler (Eds.), The Invulnerable Child (pp. 315-360). New York: The Guildford Press. Cit. Par Becoña, op. cit.

Aparicio, M. (2005). Les facteurs psychosociaux en relation avec la réussite universitaire et professionnelle. 2e thèse de doctorat. Paris : Université René Descartes, Sorbonne.

Aparicio, M. (2007a). Les facteurs psychosociaux à la base de la réussite universitaire et professionnelle : aspects psychologiques et organisationnels. HDR en Psychologie. Lille : Université de Lille3.

Aparicio, M. (2007b). Mobilité et réussite universitaires et professionnelles. Du niveau macro au niveau micro. HDR en Sciences de l'éducation. Paris : Université Paris X, Nanterre.

Aparicio, M. (2007a). Trayectorias universitarias. Un análisis a la luz de metodologías cuantitativas. Proyecto institucional, Universidad Nacional de Cuyo. Versión restringida. Tomo I. Mendoza: ZETA, $294 \mathrm{pp}$.

Aparicio, M. (2007b). Trayectorias universitarias: Un análisis a la luz de metodologías cualitativas Proyecto institucional. Universidad Nacional de Cuyo, versión restringida. Tome II. Mendoza: ZETA, $438 \mathrm{pp}$.

Aparicio, M. (2008). Causas de la Deserción en Universidades Nacionales. San Juan: Edit. UNSJ.

Aparicio, M. (2009a). La demora en los estudios universitarios. Causas desde una perspectiva cuantitativa. Tomo I. Mendoza: EDIUNC.

Aparicio, M. (2009b). La demora en los estudios universitarios. Causas desde una perspectiva cualitativa. Universidad Nacional de Cuyo, Tomo II. Mendoza: EDIUNC.

Aparicio, M. (2009 c). Les facteurs psychosociaux et la réussite universitaire et professionnelle. ANRT. Presses de l'ANRT, Lille, 469 pp et 126 pp. d'Annexes.

Aparicio, M. (2014). University Drop-Outs. A Systemic Play of Subjects, Institutions and Macro Contexts. Journal of Educational and Social Research, 4,2.

Aparicio, M. (2015 a). Towards a sui generis systemic theory: The Three-Dimensional Spiral of Sense. A Study in Argentina Applied to Identity and Professionalisation (Article 1). Asian Academic Research Journal of Social Sciences \& Humanities (AARJSH), 2(7): 246-282.

Aparicio, M. (2015 b). The Theory of the Three-Dimensional Spiral of Sense: An Application with special Reference to Identity and Professionalisation in other Disciplinary Areas (Article 2) Asian Academic Research Journal of Social Sciences \& Humanities (AARJSH), 2(7): 194-245. Aparicio, M. (2016a). Les facteurs psychosociaux à la base de la réussite universitaire et professionnelle. Aspects 
psychologiques et organisationnels. Editions Universitaires Européennes. OmniScriptum GmbH \& Co.KG., Dusseldorf, Germany. 481 pp.

Aparicio, M. (2016 b). Resiliency, Professionalization and Identity. A Study in Relation to Achievement at University at the Light of a New Paradigm: The Spiral Three Dimensional of Sens. European Journal of Interdisciplinary Studies (EJIS), Jan-Apr., 1(3): 126-131.

Aparicio, M. (2016 c). Resiliency, Professionalization and Identity. A Study in Relation to Achievement at University at the Light of a New Paradigm: The Spiral Three Dimensional of Sens. European Journal of Interdisciplinary Studies (EJIS), Jan-Apr., vol 1(3): 126-131.

Aparicio, M. (2018). «Resiliency and Cooperation or Regarding Social and Collective Competencies for University Achievement. An Analysis from a Systemic Perspective ». European Journal of Social Science Education and Research, [S.1.], 5(3) : 123-135.

Barnard, C. P. (1994). «Resiliency: A Shift in Our Perception?» American Journal of Family Theraphy, 22, 135-144.

Bandura, A. (María Zaplana, trad.) (1987). Pensamiento y acción: Fundamentos sociales. Barcelona: Martínez Roca.

Bandura, A. (1977).Self-efficacy:Toward a Unifying Theory of Behavioral Change.Psychological Review, 84 : 225-239.

Bandura, A. (1982). Self-efficacy Mechanism in Human Agency. American Psychologist, 32 : 122148.

Becoña, E. (2006). "Resiliencia: definición, características y utilidad del concepto. Revista de Psicopatología y Psicología Clínica", 11(3): 125-146.

Bronfenbrenner, U. (1981) On Making Human Beings Human. Sage Publications Ltd. Ct. In Bronfenbrenner, 1987, op.cit.

Bronfenbrenner, U. (1987). La ecología del desarrollo humano. Madrid: Paidós.

Manciaux, M., Vanistendael, S., Lecomte, J. \& Cyrulnik, B. (2001). La resiliencia: estado de la cuestión. In M. Manciaux (Ed.), La resiliencia: resistir y rehacerse. Madrid: Gedisa, 2003.

Cyrulnik, B. et al. (2004). El realismo de la esperanza. Testimonios de experiencias profesionales en torno a la resiliencia. Barcelona: Gedisa.

Cyrulnik, B. (2001). La maravilla del dolor: el sentido de la resiliencia. Barcelona: Granica.

Cyrulnik, B. (2002). Los patitos feos. La resiliencia: una infancia infeliz no determina la vida. Barcelona: Gedisa.

Coronado Hijón, A. (2017). Alumnado académicamente resiliente. In A.S. Jiménez Hernández (Ed.), Reconstruyendo un mundo con ojos de niñas (pp. 2806-2815). Granada: Editorial GEU.

Cuervo Rodríguez, J; Yanguma, C. \& Arroyave, M. (2011). Comprensiones de la resiliencia en los libros editados en español y localizados en seis bibliotecas de Bogotá, Colombia, Diversitas, 7(1): 57.

Diccionario de la Lengua Española (2005). 1 $^{\text {a }}$ ed. Madrid: Santillana.

Dyer, J. \& Minton McGuinness, T. (1996). Archives of Psychiatric Nursing, 10(5) : 276-282.

Dubs, R. (2005). "Permanecer o desertar de los estudios de postgrado: síntesis de modelos teóricos", Investigación y Postgrado, 20 (1): 55-79.

Feather, N. T., \& Davenport, P. R. (1981). Unemployment and depressive affect: A Motivational and Attributional Analysis. Journal of Personality and Social Psychology, 41(3) : 422-436

Lazarus, R.S. \& Folkman, S. (1986). Estrés y procesos cognitivos. Barcelona: Martínez Roca.

Folkman, S. \& Lazarus, R.S. (1980). "An Analysis of Coping in Middle Aged Community Sample». Journal of Health and Social Behavior, 21: 219-239.

Frydenberg, E. \& Lewis, R. (1996). ACS. Escalas de Afrontamiento para Adolescentes. Adaptation J. Pereña \& N. Seisdedos. Madrid: TEA. 
García Trujillo, S. (2011). "Resiliencia: ¿Proceso o capacidad? Una lectura crítica del concepto de resiliencia en 14 universidades colombianas". Revista Iberoamericana de Psicología, Ciencia y Tecnología, 1(4).

García-Vesga, M. C. \& Domínguez-de la Ossa, E. (2013). "Desarrollo teórico de la resiliencia y su aplicación en situaciones adversas: una revisión analítica". Revista Latinoamericana de Ciencias Sociales, Niñez y Juventud, 11 (1) ene-jun.

Gaxiola Romero, J.C.; González Lugo, S.; Domínguez Guedea, M., E. (2013). “Autorregulación, metas y rendimiento académico en bachilleres con disposiciones resilientes y no resilientes", Revista Interamericana de Psicología (Interamerican Journal of Psychology), 47 (1): 71-81.

González-Arratia, N. I. \& Valdez, J. L. (2013). Optimismo-pesimismo y resiliencia en adolescentes de una universidad pública. CIENCIA ergo-sum, 19(3):207-214.

Grotberg, E. (1995). The Internacional Resilience Proyect: Promoting Resilience in Children.

Grotberg, E. (2002). Nuevas tendencias en resiliencia. In A. Melillo, A. \& E. Suarez Ojeda. Resiliencia. Descubriendo las propias fortalezas, Buenos Aires. Paidós.

Grotberg, E. (2006). La resiliencia en el mundo de hoy. España: Gedisa.

Henderson, N. \& Milstein, M. (2003). Resiliencia en la escuela. Buenos Aires: Paidós.

Hernández, A. (1998). Familia, ciclo vital y psicoterapia sistémica breve. Bogotá: El Búho.

Kotliarenco, M.A.; Cáceres, D \& Fontecilla; D. (1997). Estado de Arte en Resiliencia. Organización Panamericana de la Salud Oficina Sanitaria Panamericana, Oficina Regional de la Organización Mundial de la Salud. CEANIM Centro de Estudios y Atención del Niño y la Mujer, Julio, Fundación W. K. Kellogg, Agencia Sueca de Cooperación Internacional para el Desarrollo.

L'American Heritage Dictionary (1994). 3hird Edition by Houghton Mifflin Company.

Lazarus, R.S. \& Folkman, S (1986). Estrés y procesos cognitivos. Barcelona: Martínez Roca.

Lorenzo, R. (2010). Nuestra capacidad de recuperación ante los obstáculos. Buenos Aires: Andrómeda.

Luthar, S. (2006). "Resilience in development: A Synthesis of Research across Five Decades". Cit por Becoña, E. (2006). Resiliencia: definición, características y utilidad del concepto, Revista de Psicopatología y Psicología Clínica, 11(3): 125-146,

Luthar, S. (1993). «Methodological an Conceptual Issues in Research on Childhood Resilence. Journal of Child Psychology and Psychiatry, 34 : 441-453.

Luthar, S.S., Ciccheti, D. \& Becker, B. (2000a). Research on Resilience: Response to commentaries. Child Development, 71: 573-575.

Luthar, S.S., Cicchetti, D. \&y Becker, B. (2000b). "The Construct of Resilience. A Critical Evaluation and Guidelines for Future Work ». Child Development, 71 : 543-562.

McClelland, D. (1960) The Achieving Society, The Free Press, 1961.

Manciaux, Vanistendael, Lecomte \& Cyrulnik (2001). La resiliencia: estado de la cuestión. Cit. par M. Manciaux (2003). Resistir o rehacerse, Barcelona, Gedisa 2003.

Manciaux, M. (Ed.) (2003). La resiliencia: resistir y rehacerse. Madrid: Gedisa.

Masten, A.S. (2001). «Ordinary Magic. Resilience Processes Indevelopment». American Psychologist, 56 : 227-238. Cit. por Becoña, 2006.

Masten, A.S., Hubbard, J.J., Gest, S.D., Tellegen, A., Garmezy, N. \& Ramírez, M. (1999). Competence in the Context of Adversity: Pathways to Resilience and Mal-adaptation from Childhood to late Adolescence. Development and Psychopathology, 11 : 143-169.

Melillo, A., Suárez, E. \& Rodríguez, D. (2004). Resiliencia y subjetividad. Los ciclos de la vida. Buenos Aires: Paidós.

Melillo, A. \& Suárez, O. E. N. (2003). Resiliencia, descubriendo las propias fortalezas. Buenos Aires: Paidós. 
Montero, I. \& Alonso Tapia, J. (1992). Cuestionario Mape II. In J. Alonso Tapia. Motivación en la adolescencia. Madrid: UAM.

Munist, M., Santos, H; Kotliarenco, M.A.; Suárez Ojeda, E, Infante, F. Grotberg, E. (2008). Manual de identificación y promoción de la resiliencia en niños y adolescentes. Organización Panamericana de la Salud. Organización Mundial de la Salud, Fundación W.K. Kellogg. Autoridad Sueca para el Desarrollo Internacional (ASDI).

Novella, A. (2002). Incremento de la resiliencia luego de la aplicación de un programa de psicoterapia breve en madres adolescentes. (Tesis de Maestría). Universidad Nacional Mayor San Marcos.

Pulgar Suazo, L. (2010). Factores de Resiliencia presentes en Estudiantes de la Universidad del Bío Bío, Sede Chillán. Tesis de Maestría.

Puerta de Klinkert, M. P. (2002). Resiliencia. La estimulación del niño para enfrentar desafíos. Buenos Aires: Lumen.

Oxford English Dictionary (1933). Oxford University Press.

Real Academia de la Lengua (1964) Enciclopedia Salvat de la Ciencia y de la Tecnología, Madrid: Salvat.

Rojas, M. (2007). Factores de riesgo y protectores identificados en adolescentes consumidores de sustancias psicoactivas. Revisión y análisis del estado actual. Disponible en <www.cedr.org.pe/ebooks/riesgo> (Consulté le 15 novembre, 2019).

Rutter, M. (1985). Resilience in the Face of Adversity: Protective Factors and Resistance to Psychiatric Disorders. British Journal of Psychiatry, 147 : 598-611.

Rutter, M. ; MacDonald, H. ; Le Couteur, A. ; Harrigton, R. ; Bolton, P. \& Bailey, A. (1991). « Debate and Argument». Child Psychol. Psychiat. 32(6): 1033-1034. Edited By: Edmund Sonuga-Barke. https://doi.org/10.1111/j.1469-7610.1991.tb01929.x-

Rutter, M. (1992). Resilience: Some Conceptual Considerations. Journal of adolescent Health, 1 (14).

Saavedra Guajardo, E. \& Villalta Paucar, M. (2008). "Medición de las características resilientes, un estudio comparativo en personas entre 15 y 65 años", LIBERABIT, 14: 31-40,

Seligman, M. (1991). El optimismo es una ventaja y un placer que se adquiere. Buenos Aires: Atlántida.

Seligman, M.E.P. \& Csikszentmihalyi, M. (2000). «Positive Psychology: An Introduction». American Psychologist, 55 : 5-14.

Suarez Ojeda, E. (1993) "Resiliencia o capacidad desobreponerse a la adversidad". Medicina y sociedad, 16(3). Buenos Aires. Argentina.

Trujillo García, S. (2011). "Resiliencia: ¿Proceso o capacidad? Una lectura crítica del concepto de resiliencia en 14 universidades colombianas". Revista Iberoamericana de Psicología, Ciencia y Tecnología, 4(1).

Vanistendael, S. (1995). ¿Cómo crecer superando los percances? BICE.

Vanistendael, S. (1994). "La Resiliencia: un concepto largo tiempo ignorado", La Infancia en el Mundo. 5(3). BICE., Montevideo.

Vanistendael, S. (1994). Como crecer superando los percances: resiliencia capitalizar las fuerzas del individuo. International Catholic Child Bureau, Ginebra. Suiza.

Vanistendael, S. \& Lecomte, J. (2002). La felicidad es posible. Barcelona: Ed. Gedisa.

Vanistendael, S. (2001). La resiliencia en lo cotidiano. In: Manciaux, M. (comp.) La resiliencia: resistir y rehacerse. Madrid: Gedisa.

Vélez, A. \& López, D. (2005). "Estrategias para vencer la deserción universitaria". Educación y Educadores, 7, 177-202. 
Wagner, E. \& Chapman, M. (1992). «Development of concern for Others ». Developmental Psychology, 28, 126-136.

Wang, M. C., Haertel, G. D., \& Walberg, H. J. (1990). What influences learning? A Content Analysis of Review Literature. Journal of Education Research, 84(1) : 30-43

Wang, M.; Haertel, G.; Walberg, H.J. (1997). «Fostering Educational Resilience in Inner-City Schools ». Publication Series No. 4.

Werner, E. \& Smith, R. (1982). Vulnerable, but Invincible: A Longitudinal Study of Resilient Children and Youth. New York, McGraw-Hill.

Werner, E.E. (1989). High-Risk Children in Young Adulthood: A Longitudinal Study from Birth to 32 Years. American Journal of Orthopsychiatry, 59 :72-81.

Wolin, S. J. \& Wolin, S. (1993). The Resilient Self: How Survivors of Troubled Families Arise above Adversity. New York: Villard Books. Cit. par Henderson \& Milstein, op. cit. 
